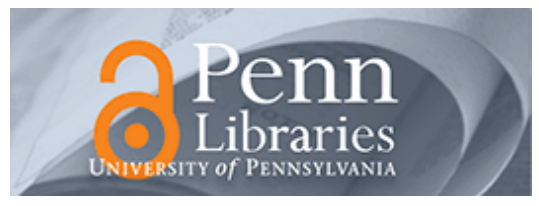

University of Pennsylvania ScholarlyCommons

$12-2015$

\title{
An Empirical Investigation of Legged Transitional Maneuvers Leveraging Raibert's Scissor Algorithm
}

Jeff Duperret

University of Pennsylvania, jdup@seas.upenn.edu

Daniel E. Koditschek

University of Pennsylvania, kod@seas.upenn.edu

Follow this and additional works at: https://repository.upenn.edu/ese_papers

Part of the Electrical and Computer Engineering Commons

\section{Recommended Citation}

Jeff Duperret and Daniel E. Koditschek, "An Empirical Investigation of Legged Transitional Maneuvers Leveraging Raibert's Scissor Algorithm", 2015 IEEE International Conference on Robotics and Biomimetics . December 2015.

This paper is posted at ScholarlyCommons. https://repository.upenn.edu/ese_papers/706

For more information, please contact repository@pobox.upenn.edu. 


\title{
An Empirical Investigation of Legged Transitional Maneuvers Leveraging Raibert's Scissor Algorithm
}

\begin{abstract}
We empirically investigate the implications of applying Raibert's Scissor algorithm to the Spring Loaded Inverted Pendulum (SLIP) model in combination with other controllers to achieve transitional maneuvers. Specifically, we are interested in how the conjectured neutral stability of Raibert's algorithm allows combined controllers to push the system's operating point around the state space without needing to expend limited control affordance in overcoming its stability or compensating for its instability. We demonstrate 2 cases where this facilitates the construction of interesting transitional controllers on a physical robot. In the first we use the motors in stance to maximize the rate of change of the body energy; in the second we take advantage of the local environmental energy landscape to push the robot's operating point to a higher or lower energy level without expending valuable motor affordance. We present data bearing on the energetic performance of these approaches in executing an accelerate-andleap maneuver on a monopedal hopping robot affixed to a boom in comparison to the cost of anchoring the robot to the SLIP template.
\end{abstract}

For more information: Kod*lab

Keywords

Agility, legged robot

Disciplines

Electrical and Computer Engineering 


\title{
An Empirical Investigation of Legged Transitional Maneuvers Leveraging Raibert's Scissor Algorithm
}

\author{
Jeffrey M. Duperret and D. E. Koditschek
}

\begin{abstract}
We empirically investigate the implications of applying Raibert's Scissor algorithm to the Spring Loaded Inverted Pendulum (SLIP) model in combination with other controllers to achieve transitional maneuvers. Specifically, we are interested in how the conjectured neutral stability of Raibert's algorithm allows combined controllers to push the system's operating point around the state space without needing to expend limited control affordance in overcoming its stability or compensating for its instability. We demonstrate 2 cases where this facilitates the construction of interesting transitional controllers on a physical robot. In the first we use the motors in stance to maximize the rate of change of the body energy; in the second we take advantage of the local environmental energy landscape to push the robot's operating point to a higher or lower energy level without expending valuable motor affordance. We present data bearing on the energetic performance of these approaches in executing an accelerate-and-leap maneuver on a monopedal hopping robot affixed to a boom in comparison to the cost of anchoring the robot to the SLIP template.
\end{abstract}

\section{INTRODUCTION}

\section{A. Transitional Maneuvers in Unstructured Environments}

Controlling a robot for transitional maneuvers over uneven terrain is a challenging task necessitated by the unstructured environment that robots must operate in, especially in search and rescue or disaster scenarios. Legged locomotion is a particularly attractive technology for traversing broken ground given the potentially versatile maneuvers afforded by multiple ground contacts, yet particularly difficult from a control perspective due to the complex dynamics involved. Several powerful control techniques with applications to legged maneuvers have been recently demonstrated that begin to allow for safe but rapid navigation of complex environments, such as model predictive control [1], reactive planning [2], geometric stabilization with little or no sensing [3], motion planning using sums-of-squares verification [4], and using the ground reaction complex to plan over different contact conditions [5]. In particular, the idea of templates and anchors [6] has proven useful in reducing complex legged dynamics to simple hybrid dynamical systems with sufficient accuracy for control strategies such as deadbeat control [7] or encoding tasks as the positive limit sets of attractive basins [8] [9]. However, deadbeat control suffers from a lack of robustness to plant uncertainty and encoding asymptotic behaviors is often not relevant to transitional behaviors that could occur over time frames as short as a single stride.

Department of Electrical and Systems Engineering, University of Pennsylvania, 200 South 33rd Street, Philadelphia, PA 19104 \{jdup,kod\}@seas.upenn.edu
While unstructured environments offer numerous challenges to the control of legged mobility, they can also offer affordances [10] that can be utilized in a control scheme to increase locomotion performance. There is an emerging view that environments act as large-scale energy landscapes that provide affordances for animal movement [11], and several studies document energetic affordances from sources of environmental compliance used by humans in athletic events [12], [13] and in tree branch compliance for a small [14] minority [15] of arboreal animals. Examples of mobile robots utilizing environmental affordances to increase specific agility [16] include passive dynamic walkers that convert gravitational energy into kinetic energy to walk without the use of motors [17], and brachiating robots using environmental "swings" to pump kinetic energy into their motions [18]. To our knowledge, efficient utilization of environmental energy affordances in combination with motors while running over non-level terrain has not been demonstrated.

\section{B. Contributions and Organization of the Paper}

In this paper we demonstrate a template based approach for controlling transitional maneuvers over non-level terrain using the Spring Loaded Inverted Pendulum (SLIP) model [19] in a way that is not deadbeat nor asymptotic, and can take advantage of the environmental energy landscape. Specifically, we corroborate empirically that Raibert's lossless Scissor Algorithm [20, Eqn 5.12] reduces the nonintegrable dynamics of SLIP to what Raibert asserted was a neutrally stable system over a subset of the state space. By applying an additional controller to the neutrally stable system we are able to push the operating point of the system around the state space without needing to expend limited control affordance overcoming any inherent stability or instability of the system.

We demonstrate that not only can the motors be used to efficiently push the neutrally stable operating point around the state space during stance to accomplish transitions, but the environment is also free to push the operating point to a higher or lower energy level, allowing the body to accumulate or dissipate kinetic energy using the external energy landscape "on the cheap." We demonstrate the efficacy of these approaches in executing an accelerate-and-leap maneuver on a monopedal hopping robot affixed to a boom.

This paper is organized as follows. In Section II we introduce the SLIP model along with Raibert's Scissor controller and conjecture that the closed loop dynamics are neutrally 
stable over a subset of the state space. In Section III we show how the neutral stability conjectured by Raibert's Scissor Algorithm is useful both in combination with other controllers and with the environment in performing transitional maneuvers. We demonstrate the utility of this approach in Section IV on a hopping monopedal robot affixed to a boom, and in Section $\mathrm{V}$ we offer conclusions and propose future work.

\section{SLIP AND RAIBERT'S SCISSOR ALGORITHM}

The SLIP template describes the dynamics of a point mass hopping on a massless spring leg. It is a relevant model for study both due to its simplicity and its ubiquity in describing legged locomotion [21]. In [20, Eqn 5.12], Raibert proposed a controller he termed the Scissor Algorithm in which the angle of the massless spring leg with respect to vertical is reflected in flight. Raibert argued (but did not formally prove) that this generates neutrally stable periodic motion in which every 2 nd stride repeats itself when forward position is discounted. Such a controller is robust to plant uncertainty because it uses only the leg angle and (Boolean) ground contact state. A full description of the closed-loop SLIP hybrid dynamics is given in Appendix I, which we refer to as the Scissor-SLIP system.

To make this more formal, let let $\mathbf{y}_{k}=\left(\mathbf{x}_{k}, \dot{\mathbf{x}}_{k}\right)=$ $\left(x_{k}, y_{k}, \theta_{\text {Leg }_{k}}, \dot{x}_{k}, \dot{y}_{k}, \dot{\theta}_{\text {Leg }, k}\right)^{T} \in \mathcal{A} \subset \mathbb{R}^{6}$ represent the apex state of SLIP before step $k$, where

$$
\left.\mathcal{A}:=\left\{(\mathbf{x}, \dot{\mathbf{x}}) \in \mathbf{T} \mathbb{R}^{3} \mid \dot{y}=0, y-l_{0} \cos \left(\theta_{\text {Leg }}\right)>0\right)\right\}
$$

and $x, y, \theta$ respectively describe the point mass forward position, relative height above the ground, and (massless) leg angle with respect to vertical which we assume is within $\Phi:=\left(-\frac{\pi}{2}, \frac{\pi}{2}\right)$ during normal operation. Let the set $\mathcal{S} \subset \mathcal{A}$ comprise apex conditions that - upon execution of a single stride of the Scissor-SLIP dynamics - achieve a subsequent apex state characterized by $\dot{y}=0$ without the mass contacting the ground, where the mapping of points in $\mathcal{S}$ to points in $\mathcal{A}$ induced by the execution of a single stride of the Scissor-SLIP dynamics is described by an apex return map $A: \mathcal{S} \rightarrow \mathcal{A}$. We conjecture that $A: \mathcal{S} \rightarrow \mathcal{S}$ and that $A$ is involutive (i.e. its double iterate returns the identity map) when forward position is projected out, making the system neutrally stable over $\mathcal{S}$, corroborating Raibert's empirical discovery [20], but a detailed formal account of these ideas lies beyond the scope of the present paper. Numerical representation of the set $\mathcal{S}$ using parameters from the monopedal hopping robot in Section IV is shown in Figure 1, indicating that $\mathcal{S}$ encompasses a large enough area of the state space to be of practicable utility on a physical platform.

We devote the remainder of this paper to examining situations in which the conjectured neutral stability afforded by the Scissor-SLIP dynamics proves useful for transitional maneuvers and demonstrate them in an experimental setting.
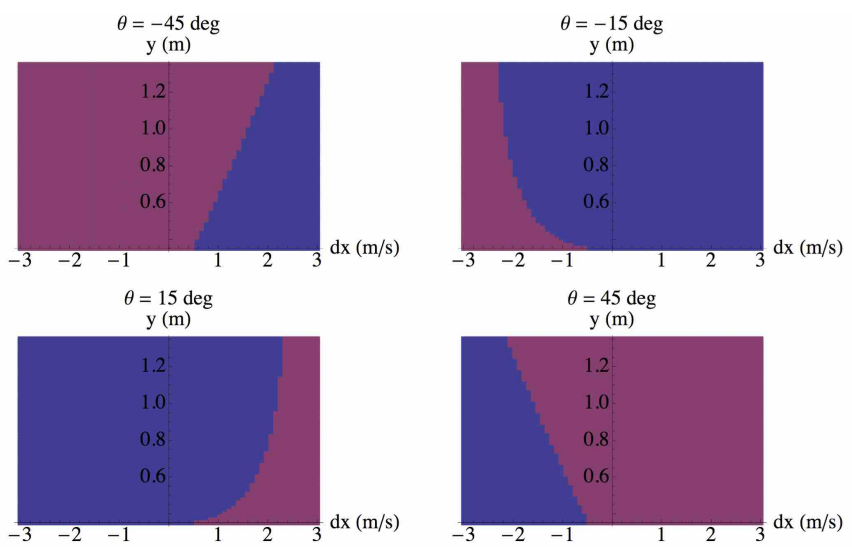

Fig. 1: Shown are slices of the $\dot{x}-y$ plane of the $\left(\dot{x}, y, \theta_{\text {Leg }}\right)$ volumetric projection of $\mathcal{S}$ (represented in blue) computed with parameters $k=5000 \frac{\mathrm{N}}{\mathrm{m}}, \mathrm{m}=1.3 \mathrm{~kg}, l_{0}=0.35 \mathrm{~m}$. The set $\mathcal{S}$ is given by the set of initial apex conditions that - upon execution of a single stride of the SLIP dynamics described in Appendix I - achieve a subsequent apex state without the mass contacting the ground.

\section{ApPliCATIONS OF THE SCISSOR-SLIP AlgORITHM: COMBINED CONTROLLERS}

The conjectured neutral stability of the Scissor-SLIP system allows the operating point of the projected apex map double iterates to be moved around the state space efficiently without needing to overcome any inherent stability or instability of the system. Efficiency is important because motor torque and power are typically limited resources and sometimes a significant portion of these resources must be devoted to anchoring the template, leaving the remaining affordance to perform the transitional maneuver. In this section we give two examples of where combining the Scissor-SLIP system with another controller can effectively utilize the energetic affordances of the environment and the actuators.

\section{A. The Height-Corrected Scissor-SLIP Controller: Utilizing the Environmental Energy Landscape}

The discrete dynamics of the Scissor-SLIP system running over uneven terrain can be represented as

$$
\mathbf{y}_{k+1}:=A\left(\mathbf{y}_{k}+\delta_{k}\right)
$$

where $\delta_{k}$ represents a change in terrain height at step $k$ and takes the form of $\delta_{k}=\left(0, \delta_{k, y}, 0,0,0,0\right)^{T}$.

We conjecture that conservation of energy holds in the Scissor-SLIP dynamics. This conjectured conservation of energy causes changes in the environmental potential energy to be "captured" by the body energy, but the Scissor-SLIP system is a closed-loop system and thus there is no available control input to choose how this accumulated (or dissipated) energy windfall manifests itself in a useful manner as relative vertical height or forward speed at the next apex.

We propose a controlled version of (2) which we call the Height-Corrected Scissor-SLIP controller (3), where the leg angle at the current apex is modified so as to affect how the 
newly accumulated (or dissipated) energy windfall affects the next apex. Let

$$
\mathbf{y}_{k+1}:=A\left(\mathbf{y}_{k}+\mathbf{u}\left(\mathbf{y}_{k}, \delta_{k}\right)+\delta_{k}\right)
$$

where $\mathbf{u}(\mathbf{y}, \delta):=(0,0, u(\mathbf{y}, \delta), 0,0,0)^{T}$ and $u: \mathcal{A} \times \mathbb{R} \rightarrow \mathbb{R}$ represents the control input at apex consisting of modifying the apex leg angle based on the state $\mathbf{y}_{k}$ and disturbance $\delta_{k}$, respectively. As the leg is massless, (3) does not violate the conjectured conservation of energy. Also we require that $\mathbf{u}\left(\mathbf{y}_{k}, 0\right)=0$ to maintain the conjectured periodicity in the absence of disturbances. Advantages of this approach include being able to implement $u(\mathbf{y}, \delta)$ on-board a robot without needing precise knowledge of the plant, and being able to use encoders alone for sensing in as described in Section IV-A.

For use on the robot in Section IV we let $u$ take the form

$$
u(\mathbf{y}, \delta)= \begin{cases}0.1 \delta & \text { if } \mathbf{y}+(0,0,0.1 \delta, 0,0,0)^{T}+\delta \in \mathcal{S} \\ \varepsilon+\theta_{\partial \mathcal{S}} & \text { else, }\end{cases}
$$

where $\theta_{\partial \mathcal{S}}$ equals the smallest magnitude choice of leg angle $\theta$ that brings $\mathbf{y}+(0,0, \theta, 0,0,0)^{T}+\delta$ into membership of the numerically computed boundary of $\mathcal{S}$, and where $\varepsilon \in \mathbb{R}$ is a small scalar whose sign is chosen such that $\mathbf{y}+\left(0,0, \varepsilon+\theta_{\partial \mathcal{S}}, 0,0,0\right)^{T}+\delta$ resides in the interior of $\mathcal{S}$. However, this particular choice of $u$ should only be viewed as an implementation detail that worked well for downhill travel in converting excess gravitational potential energy into kinetic energy at the next apex. The point is that-up to satisfying $\mathbf{y}+\mathbf{u}+\delta \in \mathcal{S}$ - the user is free to pick any form of $\mathbf{u}$ to achieve the desired behavior of their machine. Of course, as we rely on a numerically computed $\mathcal{S}$, we merely conjecture that (4) approximately achieve this.

In summary, this approach offers a way to utilize the environmental energy landscape such that changes in environment potential energy are converted into body energy using a lossless controller while maintaining periodicity in the absence of changes in terrain height. The controller can be implemented using encoders alone for sensing as described in Section IV-A.

\section{B. The Stance Energy Injection Controller: Motor Usage in Stance}

As the Scissor-SLIP dynamics represent an unactuated lossless system, the actuators of a physical robot using Raibert's Scissor algorithm are only required to anchor the template during stance. To utilize surplus actuator affordances in stance to accomplish an energetic transitional maneuver, we combine Raibert's Scissor algorithm with the following stance controller (5) that we name the Stance Energy Injection Controller.

We propose an actuated version of the SLIP stance dynamics in which we model the motors' affordance as a continuous control input $\mathbf{T}_{S}$ in stance which takes the form of an external torque generated by the motors in the Lagrangian formulation, where this torque acts through the kinematic
Jacobian according to

$$
\begin{array}{r}
\pi_{3} \circ\left(\frac{d}{d t} D_{\dot{\mathbf{q}}}-D_{\mathbf{q}}\right)\left(T_{S}(\mathbf{q}, \dot{\mathbf{q}})-V_{S}(\mathbf{q}, \dot{\mathbf{q}})\right) \\
=\left(D_{\phi} f(\phi)\right)^{-T} \mathbf{T}_{S},
\end{array}
$$

where $T_{S}$ is the kinetic energy in stance given by (8), $V_{S}$ is the potential energy in stance given by (9), $\pi_{3}: \mathbb{R}^{3} \rightarrow$ $\mathbb{R}^{2} ;\left(r, \theta, x_{\text {Toe }}\right) \mapsto(r, \theta)$ projects out the 3 rd component of the image of $\left(\frac{d}{d t} D_{\dot{\mathbf{q}}}-D_{\mathbf{q}}\right)$ as neither $x_{\text {Toe }}$ nor its derivative appear in the stance energy functions, $\mathbf{T}_{S} \in \mathbb{R}^{2}$ represents the instantaneous vector of torques asserted on their output shafts by the two independent motors, and $f$ is the forward kinematic map taking motor angles $\phi$ into $(r, \theta)$ positions of the body relative to the toe, where we assume operation in a set of states where $D_{\phi} f(\phi)$ is invertible. The map $f$ is defined in Appendix II for the specific kinematic configuration used on the robot in Section IV.

Power is commonly the limiting actuator resource in dynamic legged locomotion. However, given our interest in legged architectures using direct-drive actuation [22] where limited torque can be a significant detriment to performance, we design our stance controller assuming a torque limit instead, noting that in the experiments presented in Section IV the robot's actuators never reached their power limit in stance but frequently reached their torque limit. Hence we represent the limited motor affordance (which can be either inherent to the robot or chosen by the user) by a bound on the magnitude of the individual motor torques, i.e.,

Assumption 1: $\left\|\mathbf{T}_{S}\right\|_{\infty} \leq \lambda$ for $\lambda \in \mathbb{R}^{+}$

We actuate the motors during stance according to

$$
\mathbf{T}_{S}=\frac{\lambda}{\|\dot{\phi}\|_{\infty}} \dot{\phi}
$$

so long as the robot is in motion (such that $\dot{\phi} \neq \mathbf{0}$ ) to maximize the instantaneous rate of change of energy in stance subject to Assumption 1 (a related 1-D controller was proposed in [23] using active damping). We call this controller the Stance Energy Injection Controller and note that it can be implemented using encoders alone for sensing and without precise knowledge of the plant other than the kinematics. When used with Raibert's Scissor algorithm, it offers a way to move the neutrally stable operating point of the system around the state space. Work in presently in progress seeks to establish conditions on this controller and its domain of application guaranteeing that applying it will not cause the robot to fall over. For purposes of the present empirical paper, it suffices to observe that there were no such observed failures throughout the course of the physical experiments reported in Section IV.

\section{EXPERIMENTS}

The controllers of Section III were implemented in combination with the Scissor Algorithm on a hopping monopedal robot affixed to a boom to perform an accelerate-and-leap maneuver. We demonstrate that the conjectured neutral stability afforded by the Scissor-SLIP system allows the robot to convert potential energy accrued from running down a 
ramp into useful kinetic energy using the combined HeightCorrected Scissor-SLIP controller, and that the motors in stance can also be used to vary the operating point as shown with the Stance Energy Injection Controller to instantaneously maximizes power. The cost of anchoring the robot to SLIP is also examined.

\section{A. Experimental Setup}

1) Hardware and Experiment Description: The robot (shown in Figure 2) is a $1.3 \mathrm{~kg}$ direct-drive hopper using 2 parallel motors to locomote in the sagittal plane while torsion springs on the knees (in parallel with the motors) provide compliance with which to store potential energy during stance. Placing the springs in parallel with the motors allowed the leg kinematics to be sensed using only the motor encoders, and while a series spring could have been used instead this would likely have necessitated additional sensors for estimating the system state in stance since the spring would decouple the motors from the toe. The robot was fixed to a boom such that the body was constrained to the sagittal plane and prevented from pitching, leaving the body unconstrained in the horizontal and vertical directions. The leg configuration was chosen to be a co-planar version of [24] - both to have access to the specific agility benefit conferred by the "knee-up" configuration and to gain the simplicity of the co-planar motor packaging. The kinematic model of this leg configuration is presented in Appendix II. The upper and lower link lengths were chosen to be $0.15 \mathrm{~m}$ and $0.3 \mathrm{~m}$, respectively, and torsion springs on the knee induce a leg rest length of $0.375 \mathrm{~m}$. The control algorithms were implemented on-board the machine via an STM32-F3 microcontroller commanding custom built motor controllers [25] to drive two brushless T-Motor U8-16 100kv motors. Power was supplied by an off-board 3-cell LiPo battery. Encoders in the boom were used to estimate the energetics of the run.

Three types of experiments were performed, each one 10 times to verify repeatability. In the first experiment, the robot used both the Height-Corrected Scissor Controller ${ }^{1}$ and the Stance Energy Injection Controller to accelerate from rest over level ground, with the last recorded stride counting as the "leap" in Table I. As the terrain offered no significant source of gravitational potential energy, this experiment was used to estimate the energetic contribution of the motors in stance via the Stance Energy Injection Controller ${ }^{2}$. In the second experiment, the robot was released at the top of

\footnotetext{
${ }^{1}$ When implementing the Height-Corrected Scissor Controller, both the apex state $\mathbf{y}_{k}$ and height disturbance $\delta_{k, y}$ from (3) were not directly measured. The apex state was estimated by calculating the forward flow of the robot's liftoff state on the flight vector field (7) by direct integration, and the liftoff state was measured using encoder data. The height disturbance was reactively accounted for using the geometric encoding technique presented in [3].

${ }^{2}$ The reader may wonder why the Scissor Algorithm was used when estimating the energetic contribution of the Stance Energy Injection Controller. The reason is that without repositioning the leg in flight, it was empirically observed that the robot was all but guaranteed to fall over during the next stance event or series of stance events when only using the Stance Energy Injection Controller.
}

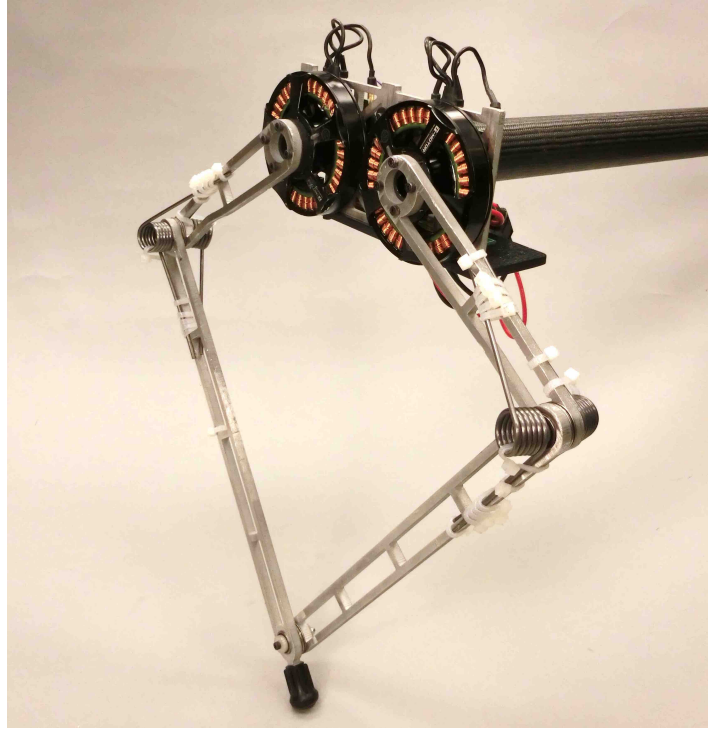

Fig. 2: The control behaviors were demonstrated on a hopping robot fixed to a boom, constraining the body in all degrees of freedom but the horizontal and the vertical.

an 18 degree ramp using only the Height-Corrected Scissor Controller where it hopped down over the course of 3 strides to build up speed, reaching level ground and leaping on the 4th stride. This experiment was done to estimate the energetic contribution of the harvested gravitational potential energy. In the third experiment, the robot was run on the ramp using both controllers to examine their combined effect over an environment offering a gravitational potential energy affordance. In this experiment the robot hopped down over the course of 2 strides (due to its higher speed as compared with the second experiment) and leapt from level ground on the 3rd stride.

2) Anchoring Description and Cost: To implement the Height-Corrected Scissor Controller and the Stance Energy Injection Controller, the robot first needed to anchor the SLIP template. This task was simplified by the fact that the robot was a monoped with a lightweight spring leg. Drop tests of the robot without the motors from heights similar to those in the experiments resulted in the robot achieving an average of 63 percent of its initial height on the next apex, indicating significant frictional losses. The SLIP anchoring thus consisted of commanding motor torques $\mathbf{T}_{A}$ to implement a virtual radial linear spring with active radial linear damping to offset frictional losses according to

$$
\mathbf{T}_{A}=\operatorname{Sat} \circ\left(D_{\phi} f(\phi)\right)^{T}\left(\begin{array}{c}
k_{p}\left(l_{0}-r\right)+k_{d} \dot{r} \\
0
\end{array}\right),
$$

where $\operatorname{Sat}()$ bounds the sup norm of $\mathbf{T}_{A}$ according to user set limits, which are specified in (6), and $k_{p}\left(l_{0}-r\right)+k_{d} \dot{r}$ represents the desired radial force of the leg subject to actuator limitations. Here $f$ is the forward kinematics map taking motor angles $\phi$ into stance body positions $(r, \theta)$ relative to the toe as defined in Appendix II, and $k_{p}, k_{d} \in \mathbb{R}^{+}$ are user chosen parameters representing the virtual linear 
TABLE I: Mean statistics for each leaping experiment

\begin{tabular}{|c|c|c|c|c|c|}
\hline \multicolumn{6}{|c|}{ Mean statistics of the hopper experiments } \\
\hline Experiment & $\begin{array}{l}\text { Leap } \\
\text { energy } \\
(\mathbf{J})\end{array}$ & $\begin{array}{l}\text { Starting } \\
\text { Energy } \\
\text { (J) }\end{array}$ & $\begin{array}{l}\text { Net } \\
\text { energy } \\
\text { gain }(\mathbf{J})\end{array}$ & $\begin{array}{l}\text { Leap } \\
\text { forward } \\
\text { speed } \\
(\mathrm{m} / \mathrm{s})\end{array}$ & $\begin{array}{l}\text { Leap } \\
\text { distance } \\
(\mathrm{m})\end{array}$ \\
\hline I & $\begin{array}{l}7.2 \\
\pm 0.1\end{array}$ & $\begin{array}{l}5.6 \\
\pm 0.1\end{array}$ & $\begin{array}{l}1.6 \\
\pm 0.1\end{array}$ & $\begin{array}{l}1.9 \\
\pm 0.1\end{array}$ & $\begin{array}{l}0.92 \\
\pm 0.05\end{array}$ \\
\hline II & $\begin{array}{l}7.6 \\
\pm 0.2\end{array}$ & $\begin{array}{l}10.8 \\
\pm 0.1\end{array}$ & $\begin{array}{l}-3.2 \\
\pm 0.2\end{array}$ & $\begin{array}{l}1.3 \\
\pm 0.2\end{array}$ & $\begin{array}{l}0.78 \\
\pm 0.05\end{array}$ \\
\hline III & $\begin{array}{l}9.4 \\
\pm 0.3\end{array}$ & $\begin{array}{l}10.8 \\
\pm 0.2\end{array}$ & $\begin{array}{l}-1.4 \\
\pm 0.3\end{array}$ & $\begin{array}{l}2.6 \\
\pm 0.1\end{array}$ & $\begin{array}{l}1.3 \\
\pm 0.04\end{array}$ \\
\hline
\end{tabular}

The mean statistics for each leaping experiment are shown in the table above. Experiment I indicates that the motors contributed on average 1.6J of energy to a leap using the Stance Energy Injection Controller, as there was no potential energy landscape with which the Height-Corrected Scissor Controller could accumulate energy. As the ramp afforded 5.2J of gravitational potential energy and in Experiment II the average net energy loss was 3.2J, Experiment II indicates that the Height-Corrected Scissor Controller was able to convert on average $2.0 \mathrm{~J}$ of the $5.2 \mathrm{~J}$ of gravitational potential energy afforded by the ramp into useful energy for a leap. Similarly, as the robot lost an average of $1.4 \mathrm{~J}$ of energy in Experiment III, we infer that the combined control scheme used in Experiment III was able to add to the body energy the $3.8 \mathrm{~J}$ difference between available gravitational energy and the lost energy. As the combined scheme of Experiment III adds 3.8J and the Stance Energy Injection Controller of Experiment I and Height-Corrected Scissor Controller of Experiment II add 1.6J and 2.0J, respectively, we deduce that the energetic contribution of the two individual controllers was approximately additive when combined in Experiment III

spring coefficient and virtual linear damping coefficient, respectively.

The anchored system was capable of being dropped from a variety of heights and maintaining a steady-state hopping behavior, however we were only able to achieve an acceptable level of performance after allocating $80 \%$ of the total torque budget in stance to the anchoring, which only left $20 \%$ of the torque budget in stance for implementing the Stance Energy Injection Controller. These allocations were enforced in software by limiting the maximum magnitude of torque each controller could apply, such that the torque $\mathbf{T}$ commanded by the motors during stance was given by

$$
\begin{array}{r}
\mathbf{T}=\mathbf{T}_{A}+\mathbf{T}_{S} \\
\text { s.t. }\left\|\mathbf{T}_{A}\right\|_{\infty} \leq 0.8 \lambda \\
\quad\left\|\mathbf{T}_{S}\right\|_{\infty} \leq 0.2 \lambda
\end{array}
$$

for a torque budget represented by a limit on the individual motor torque magnitude of $\lambda$, which was chosen to be 70 percent of the maximum torque output the motor controllers were capable of in an attempt to limit the maximum energy the robot reached during the runs so as to prevent the leg springs from bottoming out. Further details regarding the leg springs bottoming out are given in Section IV-B.

\section{B. Experimental Results}

Estimates of the apex states for the experiments are shown in Figure 3 and the mean statistics for each experiment type are shown in Table I.
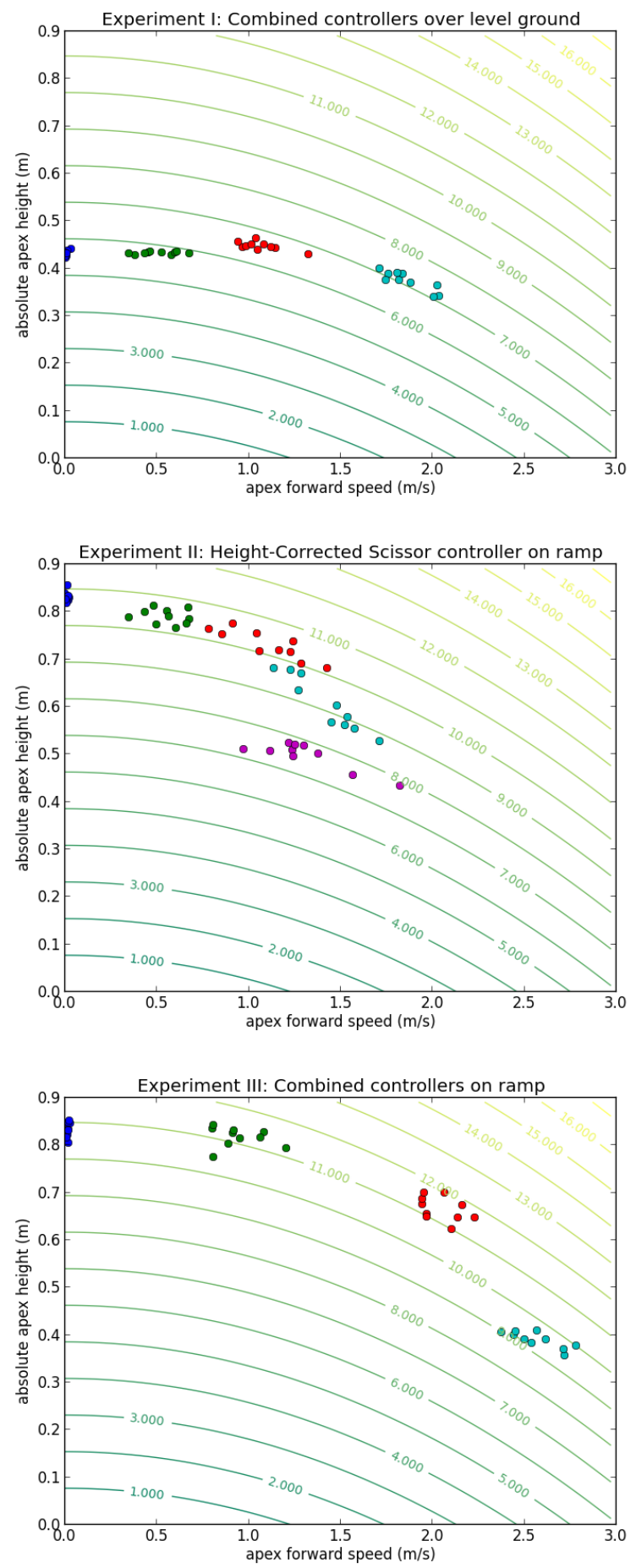

Fig. 3: The apex state for each stride in the leaping experiments is shown above, overlaid on contours showing the energy level sets in Joules. A total of 10 runs were recorded for each experiment. The first apex is given in blue, the second in green, the third in red, and the fourth in turquoise. An additional stride whose apex is shown in purple was required to clear the ramp using only the Height-Corrected Scissor Controller due to the robot's slower speed. The leap occurred in the final stride for each experiment. 
Reading from the first row of the table, for Experiment I when both controllers were used over level ground, the robot was able to leap an average of $0.9 \mathrm{~m}$ (2.4 times its nominal hip height of $0.375 \mathrm{~m}$ ) with an energy of $7.2 \mathrm{~J}-\mathrm{a} 1.6 \mathrm{~J}$ net gain from the starting energy. Reading from the second row of the table, for Experiment II, with only the HeightCorrected Scissor Controller on the ramp, the robot was able to leap an average of $0.8 \mathrm{~m}$ (2.1 times its nominal hip height of $0.375 \mathrm{~m}$ ) with an energy of $7.6 \mathrm{~J}$ - a $3.2 \mathrm{~J}$ net decrease from the starting energy, indicating that the conversion of gravitational potential energy into kinetic energy is a lossy process. The middle plot of Figure 3 shows that these inefficiencies occur primarily in the 4th stance phase when the torsion springs bottom out - when the springs compress to the point where the leg link mechanisms constraining each end collide, causing an impact that results in internal work and a loss of energy. The bottom plot of Figure 3 shows that this also occurs with the combined control scheme in Experiment III. It is likely that stiffer springs could improve the energetic performance of the machine for this task by preventing bottoming out, however this could come at the expense of decreasing the motors' ability to move the toe through the workspace. Mitigating collision losses and using more efficient springs would also increase performance, as a fundamental limit to utilizing environmental energy sources is the efficiency of repurposing it.

As the robot gained an average of 1.6J of energy in Experiment I, we infer that the Stance Energy Injection Controller was able to add an average of $1.6 \mathrm{~J}$ to the body energy as there was no potential energy landscape with which the HeightCorrected Scissor Controller could accumulate energy. The difference in starting energy shown in column 3 of the table between the robot on the ramp in Experiments II and III (a starting energy of $10.8 \mathrm{~J}$ ), and the starting energy of the robot on level ground (a starting energy of 5.6J), was 5.2J. Thus we assume that the gravitational potential energy afforded by the ramp was 5.2J. As the robot lost an average of 3.2J of energy in Experiment II, we infer that the Height-Corrected Scissor Controller was able to add to the body energy the $2.0 \mathrm{~J}$ difference between available gravitational energy and the lost energy. Of the 5.2J of initial gravitational potential energy available to the Height-Corrected Scissor Controller, a re-purposing of 2.0J gives an apparent cumulative efficiency ${ }^{3}$ of $38 \%$ over the course of 4 strides. Similarly, as the robot lost an average of 1.4J of energy in Experiment III, we infer that the combined control scheme used in Experiment III was able to add to the body energy the $3.8 \mathrm{~J}$ difference between available gravitational energy of 5.2J and the lost energy. The average energetic contribution of the Stance Energy Injection Controller in Experiment I (1.6J) and the HeightCorrected Scissor Controller of Experiment II (2.0J) sum to $3.6 \mathrm{~J}$, which is close to the $3.8 \mathrm{~J}$ of the combined control scheme of Experiment III.

An advantage of this control scheme is that the indi-

\footnotetext{
${ }^{3}$ As the motors behaved like a virtual spring and an active damper, the true energy efficiency is lower and difficult to measure. As stated previously, stiffer springs to prevent bottoming out would likely increase this efficiency.
}

vidual controllers are not fighting each other energetically as indicated by the approximate additive energetic benefit from each one. Additionally, the capability of the HeightCorrected Scissor Controller to recruit body energy from the environmental energy landscape was shown to be significant as compared to the capability of the motors using the Stance Energy Injection Controller to recruit body energy from the batteries, given the severe Stance Energy Injection Controller stance motor torque constraints we found necessary to administer so as to anchor the robot to SLIP. Analysis now in progress aims to better understand the nature and extent of the coupling between these controllers and its effect on the resulting dynamical behavior.

\section{CONClusions And Future Work}

Raibert argued (but did not formally prove) that his Scissor Algorithm confers neutrally stable closed loop dynamics to the complicated non-integrable open loop dynamics of SLIP. We empirically investigate the implications of this conjectured neutral stability in combination with other controllers to achieve transitional maneuvers. Specifically, we are interested in how Raibert's lossless Scissor Algorithm allows combined controllers to push the system's operating point around the state space without needing to expend limited control affordance overcoming any inherent stability or instability of the system.

We demonstrated 2 cases where this facilitates the construction of interesting transitional controllers on a physical robot - in one case we constructed a controller that uses the motors in stance to maximize the rate of change of the body energy, and in another case we constructed a controller that takes advantage of the environmental energy landscape to push the operating point to a higher or lower energy level "on the cheap" without expending valuable motor affordance, of which we found necessary to devote the majority to anchoring the robot to the SLIP template.

Future work now in progress addresses some of the formal questions arising from these empirical results with the hope that their clarification may yield more general approaches for transitional maneuvers and recruiting the environmental energy landscape.

\section{ACKNOWLEDGMENT}

This work is supported by the National Science Foundation Graduate Research Fellowship under Grant No. DGE0822 and by the Army Research Laboratory under Cooperative Agreement Number W911NF-10-2-0016. We would like to thank Ben Kramer for help with hopper experiments and performing numerical studies on the Scissor Algorithm.

\section{APPENDIX I}

\section{SCISSOR-SLIP HYBRID SYSTEM REPRESENTATION}

Following the notation of [26], let us represent the hybrid dynamics of SLIP [19] augmented with Raibert's scissor symmetry (where the leg is reflected at apex - as the toe is massless this does not violate the Hamiltonian constraint) [20, Eqn 5.12] as a hybrid dynamical system $\mathcal{H}=$ $(\mathcal{J}, \mathcal{K}, \mathcal{D}, \mathcal{F}, \mathcal{G}, \mathcal{R})$, where: 
- $\mathcal{J}=\{S, F\}$ represents the discrete states of stance and flight.

- $\mathcal{K}=\{(S, F),(F, S),(F, F)\}$ represents the set of discrete transitions.

- $\mathcal{D}=\mathcal{D}_{S} \coprod \mathcal{D}_{F}$ is the collection of domains, where $\mathcal{D}_{S}=\mathbf{T}\left(\left(\mathbb{R}^{2}-\{0\}\right) \times \mathbb{R}\right)$ and $\mathcal{D}_{F}=\mathbf{T} \mathbb{R}^{3}$. Specifically, let $\mathbf{q}=\left(r, \theta, x_{\mathrm{Toe}}\right)$, where $(r, \theta)$ are polar coordinates on $\mathbb{R}^{2}-\{0\}$ such that $\mathbf{T q}=(\mathbf{q}, \dot{\mathbf{q}})$ are coordinates on $\mathcal{D}_{S}$, and $\mathbf{x}=\left(x, y, \theta_{\text {Leg }}\right)$ be coordinates on $\mathbb{R}^{3}$ such that $\mathbf{T} \mathbf{x}=(\mathbf{x}, \dot{\mathbf{x}})$ are coordinates on $\mathcal{D}_{F}$. During stance the variables $r, \theta, x_{\text {Toe }}$ represent the leg length, the leg angle with respect to vertical, and the horizontal position of the toe, respectively, while during flight the variables $x, y, \theta_{\text {Leg }}$ represent the horizontal position of the mass center, the vertical position of the mass center with respect to the ground, and the leg angle with respect to vertical, respectively.

- $\mathcal{F}: \mathcal{D} \rightarrow \mathbf{T D}$, which restricts to vector fields $F_{S}$ : $\mathcal{D}_{S} \rightarrow \mathbf{T} \mathcal{D}_{S}$ on $\mathcal{D}_{S}$ and $F_{F}: \mathcal{D}_{F} \rightarrow \mathbf{T} \mathcal{D}_{F}$ on $\mathcal{D}_{F}$ such that:

$$
F_{S}(\mathbf{q}, \dot{\mathbf{q}}):=\left(\begin{array}{c}
\dot{r} \\
\dot{\theta} \\
\dot{x}_{\mathrm{Toe}} \\
-g \cos (\theta)+\frac{k}{m}\left(l_{0}-r\right)+r \dot{\theta}^{2} \\
\frac{g}{r} \sin (\theta)-\frac{2}{r} \dot{r} \dot{\theta} \\
0
\end{array}\right),
$$

where $g, k, m, l_{0} \in \mathbb{R}$ and

$$
F_{F}(\mathbf{x}, \dot{\mathbf{x}}):=\left(\begin{array}{c}
\dot{x} \\
\dot{y} \\
\dot{\theta}_{\mathrm{Leg}} \\
0 \\
-g \\
0
\end{array}\right)
$$

The parameters $g, k, m, l_{0}$ represent the acceleration due to gravity, the Hooke's law spring constant of the leg spring, the mass of the point-mass body, and the rest length of the leg, respectively.

As these vector fields describe Hamiltonian dynamics it will serve us to write the energy functions on each domain. The kinetic energy $T_{S}$ and potential energy $V_{S}$ of a state $(\mathbf{q}, \dot{\mathbf{q}}) \in \mathcal{D}_{S}$ given by

$$
\begin{gathered}
T_{S}(\mathbf{q}, \dot{\mathbf{q}}):=\frac{m}{2}\left(\dot{r}^{2}+r^{2} \dot{\theta}^{2}\right), \\
V_{S}(\mathbf{q}, \dot{\mathbf{q}}):=m g r \cos (\theta)+\frac{k}{2}\left(r-l_{0}\right)^{2},
\end{gathered}
$$

such that the total energy of a state $(\mathbf{q}, \dot{\mathbf{q}}) \in \mathcal{D}_{S}$ is given by

$$
\eta_{S}(\mathbf{q}, \dot{\mathbf{q}}):=T_{S}(\mathbf{q}, \dot{\mathbf{q}})+V_{S}(\mathbf{q}, \dot{\mathbf{q}}) .
$$

The kinetic energy $T_{F}$ and potential energy $V_{F}$ of a state $(\mathbf{x}, \dot{\mathbf{x}}) \in \mathcal{D}_{F}$ is given by

$$
T_{F}(\mathbf{x}, \dot{\mathbf{x}}):=\frac{m}{2}\left(\dot{x}^{2}+\dot{y}^{2}\right), \quad V_{F}(\mathbf{x}, \dot{\mathbf{x}}):=m g y
$$

such that the total energy of a state $(\mathbf{x}, \dot{\mathbf{x}}) \in \mathcal{D}_{F}$ is given by

$$
\eta_{F}(\mathbf{x}, \dot{\mathbf{x}}):=T_{F}(\mathbf{x}, \dot{\mathbf{x}})+V_{F}(\mathbf{x}, \dot{\mathbf{x}}) .
$$

- $\mathcal{G}=G_{S, F} \coprod G_{F, S} \coprod G_{F, F}$ is the collection of guards such that:

$$
\begin{gathered}
G_{S, F}:=\left\{(\mathbf{q}, \dot{\mathbf{q}}) \in \mathcal{D}_{S} \mid l_{0}-r=0, \dot{r}>0\right\}, \\
G_{F, S}:=\left\{(\mathbf{x}, \dot{\mathbf{x}}) \in \mathcal{D}_{F} \mid l_{0} \cos \left(\theta_{\mathrm{Leg}}\right)-y=0,\right. \\
\left.\frac{-l_{0} \sin \left(\theta_{\mathrm{Leg}}\right) \dot{x}+y \dot{y}}{\sqrt{l_{0}^{2} \sin \theta_{\mathrm{Leg}}{ }^{2}+y^{2}}}<0\right\},
\end{gathered}
$$

and

$$
G_{F, F}:=\left\{(\mathbf{x}, \dot{\mathbf{x}}) \in \mathcal{D}_{F} \mid \dot{y}=0, y-l_{0} \cos \left(\theta_{\text {Leg }}\right)>0\right\},
$$

where $G_{F, F}$ can be thought of as the apex of flight and is equivalent to $\mathcal{A}$ via (1).

- $\mathcal{R}: \mathcal{G} \rightarrow \mathcal{D}$ is the reset map, a continuous map that restricts to $R_{S, F}: G_{S, F} \rightarrow \mathcal{D}_{F}$ on $G_{S, F}, R_{F, S}:$ $G_{F, S} \rightarrow \mathcal{D}_{S}$ on $G_{F, S}$, and $R_{F, F}: G_{F, F} \rightarrow \mathcal{D}_{F}$ on $G_{F, F}$, where:

$$
\begin{gathered}
R_{S, F}(\mathbf{q}, \dot{\mathbf{q}}):=\left(\begin{array}{c}
x_{\mathrm{Toe}}-r \sin (\theta) \\
r \cos (\theta) \\
\theta \\
-\dot{r} \sin (\theta)-r \dot{\theta} \cos (\theta) \\
\dot{r} \cos (\theta)-r \dot{\theta} \sin (\theta) \\
0
\end{array}\right), \\
R_{F, S}(\mathbf{x}, \dot{\mathbf{x}}):=\left(\begin{array}{c}
l_{0} \\
\theta_{\text {Leg }} \\
x+r \sin (\theta) \\
\dot{y} \cos \left(\theta_{\text {Leg }}\right)-\dot{x} \sin \left(\theta_{\text {Leg }}\right) \\
-\frac{1}{l_{0}}\left(\dot{x} \cos \left(\theta_{\text {Leg }}\right)+\dot{y} \sin \left(\theta_{\text {Leg }}\right)\right) \\
0 \\
R_{F, F}(\mathbf{x}, \dot{\mathbf{x}}):=\phi_{F}^{\varepsilon}\left(x, y,-\theta_{\text {Leg }}, \dot{x}, \dot{y}, \dot{\theta}_{\text {Leg }}\right)
\end{array}\right),
\end{gathered}
$$

where $\phi_{F}^{t}$ is the flow on $\mathcal{D}_{F}$ and $\varepsilon$ is some infinitesimal positive real number such that $x \in G_{F, F} \Rightarrow R_{F, F}(x) \notin$ $G_{F, F}$.

It will provide the reader with some intuition to note that the seemingly complicated form of $G_{F, S}$ is simplified when applying $R_{F, S}$ to $G_{F, S}$, i.e.

$$
\begin{aligned}
& R_{F, S}\left(G_{F, S}\right)=\left\{(\mathbf{q}, \dot{\mathbf{q}}) \in \mathcal{D}_{S} \mid l_{0}-r=0, \dot{r}<0\right\} . \\
& \text { ApPendix II } \\
& \text { Kinematic Model of The Hopping Robot }
\end{aligned}
$$

\section{Kinematic Model of THE Hopping Robot}

A parallel linkage representation of the robot used in Section IV is shown in Figure $4 \mathrm{a}$, where the red link represents the separation of the motor shafts with distance $d=0.1 \mathrm{~m}$ from one another, the yellow links represent the upper linkages with lengths $l_{1}=0.15 \mathrm{~m}$, and the green links represent the lower linkages with lengths $l_{2}=0.3 \mathrm{~m}$. The length of the toe extending from the lower link is disregarded in this representation. 
For ease of computation on the robot's microcontroller we made the simplifying assumption that $d=0$ as shown in Figure $4 \mathrm{~b}$. The forward and inverse kinematic maps given by (11) and (12) of this kinematic model are easily calculated on-board the robot's microcontroller for real-time application and were used in implementing the controllers in the experiments of Section IV.

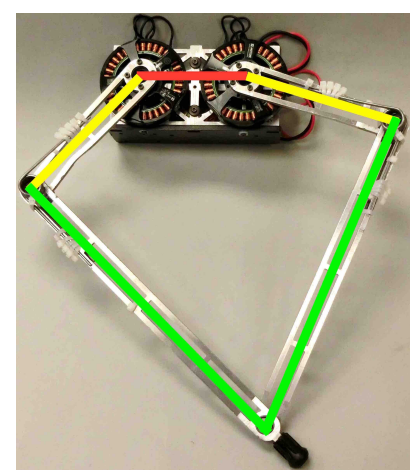

(a) Parallel linkage representation of the robot, disregarding the toe length.

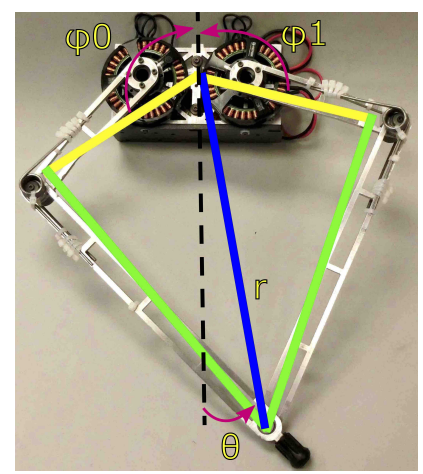

(b) Kinematic approximation to Fig. 4a, assuming co-radial motor shafts.
Fig. 4: Two parallel linkage representations of the robot kinematics. While the left is a more accurate representation of the underlying kinematics, the forward and inverse kinematics of the right can be calculated much faster on the robot's microcontroller.

The forward kinematic map $f$ and inverse kinematic map $g$ of the kinematic representation shown in Figure $4 \mathrm{~b}$ are given by:

$f\left(\phi_{0}, \phi_{1}\right):=\left[-l_{1} \cos \left(\frac{\phi_{0}+\phi_{1}}{2}\right)+\sqrt{l_{2}^{2}-l_{1}^{2} \sin ^{2}\left(\frac{\phi_{0}+\phi_{1}}{2}\right)}, \ldots\right.$

$$
g(r, \theta):=\left[\begin{array}{l}
\pi-\arccos \left(\frac{r^{2}+l_{1}^{2}-l_{2}^{2}}{2 r l_{1}}\right)+\theta \\
\pi-\arccos \left(\frac{r^{2}+l_{1}^{2}-l_{2}^{2}}{2 r l_{1}}\right)-\theta
\end{array}\right] .
$$

\section{REFERENCES}

[1] H.-W. Park, P. M. Wensing, S. Kim et al., "Online planning for autonomous running jumps over obstacles in high-speed quadrupeds," in Proceedings of the Robotics: Science and System (RSS), June 20-22 2015 , p. (to appear).

[2] Ö. Arslan and U. Saranli, "Reactive planning and control of planar spring-mass running on rough terrain," IEEE Transactions on Robotics, vol. 28, no. 3, pp. 567-579, 2012.

[3] G. Council, S. Yang, and S. Revzen, "Deadbeat control with (almost) no sensing in a hybrid model of legged locomotion," in International Conference on Advanced Mechatronic Systems, ICAMechS, 2014, pp. 475-480.

[4] R. Tedrake, I. R. Manchester, M. Tobenkin, and J. W. Roberts, "Lqrtrees: Feedback motion planning via sums-of-squares verification," International Journal of Robotics Research, vol. 29, no. 8, pp. 1038$1052,2010$.

[5] A. M. Johnson and D. E. Koditschek, "Toward a vocabulary of legged leaping," in Proceedings of the 2013 IEEE Intl. Conference on Robotics and Automation, May 2013, pp. 2553-2560.

[6] R. J. Full and D. E. Koditschek, "Templates and anchors: Neuromechanical hypotheses of legged locomotion on land," Journal of Experimental Biology, vol. 202, no. 23, pp. 3325-3332, 1999.
[7] I. Uyanik, Ö. Morgl, and U. Saranli, "Experimental validation of a feed-forward predictor for the spring-loaded inverted pendulum template," IEEE Transactions on Robotics, vol. 31, no. 1, pp. 208216, 2015.

[8] A. Degani, A. W. Long, S. Feng, H. Benjamin Brown, R. D. Gregg, H. Choset, M. T. Mason, and K. M. Lynch, "Design and openloop control of the parkourbot, a dynamic climbing robot," IEEE Transactions on Robotics, vol. 30, no. 3, pp. 705-718, 2014.

[9] R. M. Ghigliazza, R. Altendorfer, P. Holmes, and D. Koditschek, "A simply stabilized running model," SIAM Journal on Applied Dynamical Systems, vol. 2, no. 2, pp. 187-218, 2003.

[10] J. G. Greeno, "Gibson's affordances," Psychological review, vol. 101, no. 2, pp. 336-342, 1994.

[11] E. L. C. Shepard, R. P. Wilson, W. G. Rees, E. Grundy, S. A. Lambertucci, and S. B. Vosper, "Energy landscapes shape animal movement ecology," American Naturalist, vol. 182, no. 3, pp. 298312, 2013.

[12] T. A. McMahon and P. R. Greene, "The influence of track compliance on running," Journal of Biomechanics, vol. 12, no. 12, pp. 893-904, 1979.

[13] A. Arampatzis, F. Schade, and G. . Brggemann, "Effect of the polehuman body interaction on pole vaulting performance," Journal of Biomechanics, vol. 37, no. 9, pp. 1353-1360, 2004.

[14] S. K. S. Thorpe, R. H. Crompton, and R. M. Alexander, "Orangutans use compliant branches to lower the energetic cost of locomotion," Biology Letters, vol. 3, no. 3, pp. 253-256, 2007.

[15] A. J. Channon, M. M. Gnther, R. H. Crompton, K. D'Aot, H. Preuschoft, and E. E. Vereecke, "The effect of substrate compliance on the biomechanics of gibbon leaps," Journal of Experimental Biology, vol. 214, no. 4, pp. 687-696, 2011.

[16] J. M. Duperret, G. D. Kenneally, J. L. Pusey, and D. E. Koditschek, "Towards a comparative measure of legged agility," in International Symposium on Experimental Robotics, June 2014.

[17] T. McGeer, "Passive dynamic walking," International Journal of Robotics Research, vol. 9, no. 2, pp. 62-82, 1990.

[18] J. Nakanishi, T. Fukuda, and D. Koditschek, "A brachiating robot controller," IEEE Transactions on Robotics and Automation, vol. 16, no. 2, pp. 109-123, 2000.

[19] U. Saranli, W. J. Schwind, and D. E. Koditschek, Toward the control of a multi-jointed, monoped runner, 1998, vol. 3, p. 26762682.

[20] M. H. Raibert, Legged Robots That Balance. Cambridge: MIT Press, 1986.

[21] D. E. Koditschek, R. J. Full, and M. Buehler, "Mechanical aspects of legged locomotion control," Arthropod Structure and Development, vol. 33, no. 3, pp. 251-272, 2004.

[22] G. Kenneally, A. De, and D. Koditschek, Design Principles for a Family of Direct-Drive Legged Robots, Jul 2015, p. https://sites.google.com/site/miniatureleggedrobots/. [Online]. Available: https://sites.google.com/site/miniatureleggedrobots/

[23] G. Secer and U. Saranli, "Control of hopping through active virtual tuning of leg damping for serially actuated legged robots," in Proceedings - IEEE International Conference on Robotics and Automation, 2014, pp. 4556-4561.

[24] G. Kenneally and D. E. Koditschek, "Kinematic leg design in an electromechanical robot," Workshop article presented at ICRA 2014, Hong Kong, Tech. Rep., May 2014. [Online]. Available: http://kodlab.seas.upenn.edu/Kenneally/ICRA2014

[25] A. De and D. E. Koditschek, "The Penn Jerboa: A platform for exploring parallel composition of templates," http://arxiv.org/abs/1502.05347, Tech. Rep., Feb 2015, arXiv: 1502.05347.

[26] A. M. Johnson, S. E. Burden, and D. E. Koditschek, "A hybrid systems model for simple manipulation and self-manipulation systems," $\operatorname{arXiv}$ preprint arXiv:1502.01538 [cs.RO], 2015. 